\title{
Existence of outermost apparent horizons with product of spheres topology
}

\author{
FERNANDO SCHWARTZ
}

\begin{abstract}
In this paper we construct the first examples of $(n+m+2)$ dimensional asymptotically flat Riemannian manifolds with non-negative scalar curvature that have outermost minimal hypersurfaces with non-spherical topology for $n, m \geq 1$.

The outermost minimal hypersurfaces are, topologically, $S^{n} \times$ $S^{m+1}$. In the context of general relativity these hypersurfaces correspond to outermost apparent horizons of black holes.
\end{abstract}

\section{Introduction and main result}

It follows from the well-known works of Meeks, Simon and Yau [13, 14] (cf. $[11, \S 4]$ ) that (a) the outermost minimal surface of an asymptotically flat 3-dimensional Riemannian manifold with non-negative scalar curvature is, topologically, a 2-sphere and that (b) the complement of the region enclosed by the outermost minimal surface is diffeomorphic to $\mathbb{R}^{3}$ minus a finite number of balls. Galloway and Schoen generalized statement (a) to higher dimensions in $[7,8]$, where they proved that, in dimensions three and above, the outermost minimal hypersurface of an AF manifold with non-negative scalar curvature must be of positive Yamabe type, i.e., it admits a metric of positive scalar curvature.

A natural question that arises in this context is to determine whether there exist high-dimensional AF manifolds with non-negative scalar curvature having non-spherical outermost minimal hypersurfaces.

In this paper we answer the above question in the affirmative by constructing the first examples of AF manifolds with non-negative scalar curvature that have non-spherical outermost minimal hypersurfaces in dimensions 4 and above. In particular, a consequence of our work is that (b) does not hold in high dimensions. 
Our result is inspired by, although unrelated to, the recent discovery of a black ring ${ }^{1}$ in general relativity. In that context, the apparent horizon of the black hole plays the role of the outermost minimal hypersurface of an AF Riemannian manifold with non-negative scalar curvature. Our main result is the following.

Theorem 1.1. For any $n, m \geq 1$ there exists an asymptotically flat, scalar flat $(n+m+2)$-dimensional Riemannian manifold $(M, g)$ with outermost apparent horizon which is an outermost smooth minimal hypersurface with topology $S^{n} \times S^{m+1}$.

Remark 1.2. An off-shot of the construction (Corollary 5.13) is that inside the manifolds we construct there are complete non-compact minimal hypersurfaces with a conical singularity at a point. These hypersurfaces can be thought of as generalized minimal cones.

The proof of the theorem uses a fairly general procedure. We expect to be able to construct many more non-spherical topologies via this process in our forthcoming work. Our theorem also has some relevance in highdimensional relativity, since it gives suitable initial data with non-spherical apparent horizon that evolves into a black hole. This behavior is particularly interesting in high dimensions due to string theory (cf. [15]), and also because there are the only two known examples of non-spherical black holes, both 5-dimensional: Emparan and Reall's black ring [5] from above, and Elvang and Figueras' black saturn ${ }^{2}$ [4]. (In [6] approximate spacetime solutions with black ring topology $S^{1} \times S^{n}, n \geq 2$ appear.)

Overview of the proof. We construct $(M, g)$ by conformally blowing up an $n$-sphere inside $\mathbb{R}^{n+m+2}$. We show that the resulting manifold has a compact outermost apparent horizon because its ends are positive. Then, using the symmetry of the construction, ODE analysis and a standard geometric measure theory argument, we prove that the horizon of $M$ is a smooth minimal hypersurface that possibly contains a conical singularity. To prove that the horizon is actually smooth we use a maximum principle that gives that the horizon is either (1) smooth everywhere and with topology $S^{n} \times S^{m+1}$ or (2) 'close' to having (or having) a conical singularity. Case (2) is ruled out by an ODE analysis of a certain minimal surface equation, where we show

\footnotetext{
${ }^{1}$ This is a 5 -dimensional rotating black-hole spacetime with horizon topology $S^{1} \times S^{2}$.

${ }^{2} \mathrm{~A}$ black ring rotating around a spherical black hole
} 
that being close to having a conical singularity would cause the horizon to be unbounded, but this is impossible since we know it is compact.

This paper is organized as follows. In Section 2 we introduce notation and give a general argument for the existence of apparent horizons when barriers are present. In Section 3 we construct $(M, g)$. We also write a simple formula for the mean curvature of symmetric hypersurfaces in it. We use the formula to show that $(M, g)$ has barriers. In Section 4 we prove the horizon is a smooth minimal hypersurface possibly with a conical singularity at the origin. In Section 5 we show that the horizon is smooth everywhere and has topology $S^{n} \times S^{m+1}$.

\section{Preliminaries}

Let $\left(M^{n}, g\right)$ denote an $n$-dimensional Riemannian manifold with an asymptotically flat end $E$ and other ends $\left\{E_{k}\right\}$. In this paper we are interested in studying the outermost horizon of $M$ with respect to the AF end $E$. To define it, we work with $\hat{M}$, which is $M$ with the ends $\left\{E_{k}\right\}$ compactified by adding the points $\left\{\infty_{k}\right\}$.

A marginally trapped region of $\hat{M}$ (sometimes called marginally outer trapped) is an open pre-compact set $R \subset \hat{M}$ with boundary $\Sigma=\partial R$, which is a compact smooth hypersurface with non-positive mean curvature $h_{g}(\Sigma) \leq 0$. (In our convention, negative mean curvature means that the area of $\Sigma$ decreases under a variation in the outward-pointing normal direction.) Any such boundary $\Sigma$ of a marginally trapped region $R$ is called a marginally trapped hypersurface.

Let $\mathscr{R}$ denote the set of all marginally trapped regions in $\hat{M}$. Following Wald [20] we define the apparent horizon of $(M, g)$ with respect to the end $E$ as the boundary of the closure of the union of all marginally trapped regions of $\hat{M}$.

Definition 2.1. The apparent horizon of $(M, g)$ is $\Sigma^{*}=\partial(\overline{\cup \mathscr{R}})$.

The apparent horizon of $M$ is clearly unique and outermost. On the other hand, the apparent horizon is not necessarily smooth. Nevertheless, in our construction we will prove that the horizon is a smooth minimal hypersurface by virtue of symmetry and some other considerations.

A natural question that arises is to determine geometric conditions that guarantee the presence of an apparent horizon. We say that $(M, g)$ has positive ends if it has two or more ends, and each end of $M$ can be foliated 
by smooth compact hypersurfaces with positive mean curvature. A standard argument, for which we only sketch a proof, is the following.

Theorem 2.2. A manifold with positive ends has an apparent horizon.

Proof. Clearly the set $\mathscr{R}$ is non-empty since the region bounded by a union of exactly one leaf from each end (except for the non-compactified one) is marginally trapped. We claim that no marginally trapped hypersurface can enter the positive region of the non-compact end, from which it follows that $\overline{\cup \mathscr{R}} \neq M$. Indeed, suppose that a smooth compact hypersurface intersects the positive foliation of the non-compact end. At the farthest point with respect to the foliation, the hypersurface must be tangent to the foliation. By the maximum principle, the mean curvature of the hypersurface is positive at that point. Thus it is not marginally trapped.

\section{Existence}

We begin this section by constructing the manifold $(M, g)$. Our choice of metric $g$ is highly symmetric, as we see below. This makes the formula for the mean curvature of symmetric hypersurfaces easy to compute. Near the end of this section we use the mean curvature formula for symmetric hypersurfaces to show that $M$ has positive ends. The existence of an apparent horizon in $(M, g)$ follows from Theorem 2.2.

Fix $m, n \geq 1 .(M, g)$ is, basically, $(n+m+2)$-dimensional Euclidean space minus an $n$-sphere endowed with a conformally related metric that blows up on the $n$-sphere.

The construction is as follows. Consider the sphere $S^{n}=S^{n} \times\{0\}^{m+1}$ sitting inside the first $(n+1)$-dimensional factor of $\mathbb{R}^{n+m+2}$. Let $G_{p}$ denote the Green's function for the Laplacian around $p \in \mathbb{R}^{n+m+2}$, i.e., the function $G_{p}(q)=|p-q|^{-(n+m)}$, where $|p-q|$ is the Euclidean norm.

For $\epsilon>0$ (which we later require to be small) our conformal factor $U$ is the smooth positive function defined on $\mathbb{R}^{n+m+2} \backslash S^{n}$ by the formula

$$
U(p)=1+\epsilon G_{p} * \chi_{S^{n}}=1+\epsilon \int_{S^{n}}|p-q|^{-(n+m)} d \mu(q) .
$$

Definition 3.1. We define $(M, g)$ to be $\mathbb{R}^{n+m+2} \backslash S^{n}$ endowed with the conformally flat metric $g=U^{4 /(n+m)} \delta_{i j}$. (For simplicity, $\epsilon$ is removed from the definition of $g$.)

Lemma 3.2. For $(M, g)$ defined as above we have that 
(i) $g$ is $\mathrm{SO}(n+1) \times \mathrm{SO}(m+1)$ invariant, so the group acts by isometries on $(M, g)$ and fixes both ends.

(ii) $\Delta_{0} U=0$ outside $S^{n}$, so $(M, g)$ is scalar flat.

(iii) Let $r(p)=\operatorname{dist}\left(p, S^{n}\right)$. For $p$ near $S^{n}$ we have $U(p)=1+\epsilon r^{-m}+$ $O\left(r^{1-m}\right)$ whenever $m>1$. For $m=1, U(p)=1+\epsilon r^{-1}+O(\log r)$.

(iv) There exists $\alpha=\alpha(n, m)>0$ so that as $|p| \rightarrow \infty, \quad U(p)=1+\epsilon \alpha$ $|p|^{-(n+m)}+O\left(|p|^{1-(n+m)}\right)$, so spatial infinity is asymptotically flat.

Proof. Statement (i) is direct from the definition of $U$. For (ii), Green's formula gives that $U$ is harmonic. The transformation law for the scalar curvature under conformal deformations is $R_{g}=-U^{-(n+m+4) /(n+m)}(4(n+$ $\left.m+1) /(n+m) \Delta_{0} U-R_{0} U\right)$ which implies $R_{g}=0$. Statement (iii) follows from the expansion $U(p)=1+\epsilon \int_{S^{n}}\left(p_{1}^{2}+\cdots+p_{m+1}^{2}+\sum_{i=1}^{n+1}\left(p_{i+m+1}-\right.\right.$ $\left.\left.\xi_{i}\right)^{2}\right)^{-(n+m) / 2} d \mu(\xi)$, which is a particular case of the calculation in the Appendix of [16]. The asymptotic formula in (iv) holds because of the maximum principle together with $u(p) \rightarrow 1$ as $|p| \rightarrow \infty$, and $u(p) \rightarrow+\infty$ as $\operatorname{dist}\left(p, S^{n}\right) \rightarrow 0$. Standard calculations as those in [1] show that the end is asymptotically flat in this case.

The manifold $(M, g)$ is invariant under the action of action of $\mathrm{SO}(n+$ $1) \times \mathrm{SO}(m+1)$. We are interested in finding a formula for the mean curvature of $\mathrm{SO}(n+1) \times \mathrm{SO}(m+1)$-invariant hypersurfaces of $(M, g)$ since the apparent should also be invariant. In order to do that, we first find a formula for the mean curvature of $\mathrm{SO}(n+1) \times \mathrm{SO}(m+1)$-invariant hypersurfaces of Euclidean space, and then apply the transformation law for the mean curvature under conformal deformations.

Let $\Sigma \subset\left(\mathbb{R}^{n+m+2}, \delta i j\right)$ be an $\mathrm{SO}(n+1) \times \mathrm{SO}(m+1)$-invariant hypersurface. If we write a vector in $\mathbb{R}^{n+1} \times \mathbb{R}^{m+1}$ as $(x, y)$ respecting this decomposition, then $\Sigma$ has the form

$$
\Sigma(\gamma)=\{(x, y):(|x|,|y|) \in \gamma\}
$$

where $\gamma$ is some curve in the quadrant $\mathbb{R}_{+}^{2}=\{(a, b) \geq(0,0)\}$.

If $\gamma=(x(s), y(s))$ is oriented and parameterized by arc length, the principal curvatures of $\Sigma(\gamma)$ with respect to an outward pointing unit normal are $\kappa,-\dot{x} / y, \ldots,-\dot{x} / y, \dot{y} / x, \ldots, \dot{y} / x$, where $\kappa$ is the curvature of $\gamma$ with respect to the flat metric. Therefore, the mean curvature of $\Sigma$ inside Euclidean space is given by

$$
h_{0}=\kappa+n \dot{y} / x-m \dot{x} / y
$$


Example 3.3. The coordinate sphere in $\mathbb{R}^{n+m+2}$ is $S_{R}(0)=\Sigma(\gamma)$, where $\gamma=(R \cos s / R, R \sin s / R), 0 \leq s \leq \pi R / 2$. Equation (3.3) gives $h_{0}=(n+$ $m+1) / R$.

The transformation law for the mean curvature under conformal deformations gives that the mean curvature of a hypersurface $N \subset(M, g)$ is

$$
h=U^{2-c}\left(h_{0}+c \partial_{\nu} \ln U\right),
$$

where $c=2(n+m+1) /(n+m)$ and $\partial_{\nu}$ is the Euclidean outward-pointing normal derivative.

If we consider an $\mathrm{SO}(n+1) \times \mathrm{SO}(m+1)$-invariant hypersurface $\Sigma(\gamma)$ like before, and fix the orientation of $\gamma$ so that the outward-pointing normal derivative is given by $\partial_{\nu}=\dot{y} \partial_{x}-\dot{x} \partial_{y}$, then the quantity (3.4) from above is easily computed using Equation (3.3). We write this in the following result.

Lemma 3.4. Let $\gamma=(x(s), y(s))$ be parameterized by arc length and oriented as above. Then, the mean curvature of $\Sigma(\gamma) \subset(M, g)$ is

$$
h=U^{2-c}\left(\kappa+\dot{y}\left(\frac{n}{x}+c \frac{U_{x}}{U}\right)-\dot{x}\left(\frac{m}{y}+c \frac{U_{y}}{U}\right)\right),
$$

where $c=2(n+m+1) /(n+m)$. If $\gamma$ is the graph of $y(x)$, the mean curvature is given by

$$
h= \pm \frac{U^{2-c}}{\sqrt{1+y^{\prime 2}}}\left(\frac{y^{\prime \prime}}{1+y^{\prime 2}}+y^{\prime}\left(\frac{n}{x}+c \frac{U_{x}}{U}\right)-\left(\frac{m}{y}+c \frac{U_{y}}{U}\right)\right) .
$$

A discussion on minimal hypersurfaces of the Euclidean metric that are $\mathrm{SO}(n+1) \times \mathrm{SO}(m+1)$-invariant appears in [12]. Radially symmetric minimal hypersurfaces of Euclidean space have been studied in [9, 10, 19]. Singular solutions of the minimal surface equation in flat space appear in [2], where minimal cones in dimension 8 are constructed as examples of minimizers that are singular on the origin. More recent references include [3] and $[18]$.

We now use Equation (3.5) to compute the mean curvature of barriers that foliate the ends of $M$. The foliations consist of large coordinate spheres on the asymptotically flat end, and small tubes around the missing $S^{n}$ on the other end. An essential fact is that the tubes around the missing $S^{n}$ have topology $S^{n} \times S^{m+1}$ since they are of the form $\partial\left(S^{n} \times B^{m+2}\right)$.

Theorem 3.5. $M$ has positive ends. 
Proof. We begin by foliating a neighborhood of the missing $S^{n}$ by the tubes $T_{r}=\Sigma\left(\gamma_{r}\right)$, where $\gamma_{r}=(1+r \cos (s / r), r \sin (s / r))$ for $r>0$ small, $s \in$ $[0, \pi r]$. Using the asymptotics of Lemma 3.2(iii) and plugging $\gamma_{r}$ into (3.5) gives

$$
\begin{aligned}
h\left(T_{r}\right)= & -U^{2-c}\left(\frac{1}{r}\left[(m+1)-\frac{2 m(n+m+1)}{(n+m)\left(1+r^{m} / \epsilon\right)}\right]+\frac{n \cos (s / r)}{1+r \cos (s / r)}\right) \\
& + \text { lower-order terms, }
\end{aligned}
$$

which is a positive quantity for $0<r<c^{\prime} \epsilon^{1 / m}$ and $\epsilon>0$ small enough, where $c^{\prime}>0$ only depends on $n, m$. (For this calculation we reverse the sign of Equation (3.5) since the outward-pointing normal of the tubes $T_{r}$ points towards the missing sphere.)

On the other hand, the mean curvature of all large-enough coordinate spheres $S_{R}(0)=\Sigma\left(\gamma_{R}\right)$, where $\gamma_{R}=(R \cos (s / R), R \sin (s / R))$ for $t \in$ $[0, \pi R / 2]$ is positive since $M$ is asymptotically flat. Indeed, it follows from Example 3.3, Equation (3.5) and Lemma 3.2(iv) that $h\left(S_{R}\right)=O(1)((n+$ $\left.m+1) / R-\epsilon \cdot O\left(R^{-(n+m+2)}\right)\right)$. The right-hand side of this equation is positive for all $R$ large enough, and continues to be so for all small values of $\epsilon>0$.

Corollary 3.6. $M$ has an apparent horizon.

\section{Regularity}

In this section we prove that the horizon is a smooth minimal hypersurface except possibly at the origin, where it may have a conical singularity. This last option is ruled out in the next section.

Recall that given a curve $\gamma \subset \mathbb{R}_{+}^{2}$, the set $\Sigma(\gamma)$ is defined as the subset $\Sigma(\gamma)=\{(|\vec{x}|,|\vec{y}|) \in \gamma\} \subset \mathbb{R}^{n+m+2}$. We will see that the apparent horizon is given by $\Sigma\left(\gamma^{*}\right)$, where $\gamma^{*}$ is a particular curve. As a matter of fact, from what we have done so far we can already say a few things about $\gamma^{*}$, assuming this decomposition holds. For example, the horizon is contained in a compact set, therefore $\gamma^{*}$ should be contained in a bounded region. The horizon encloses the missing sphere, therefore $\gamma^{*}$ should separate $\mathbb{R}_{+}^{2}$ in two regions, one containing all the tubular barriers of Theorem 3.5. This motivates the following definition. Let $N_{\epsilon} \subset \mathbb{R}_{+}^{2}$ denote a fixed (depends only on $\epsilon$ ) semicircular marginally trapped region that is a neighborhood of $(1,0)$. (This is possible by the proof of Theorem 3.5.)

Definition 4.1. Let $\gamma:\left(-L_{1}, L_{2}\right) \rightarrow \mathbb{R}_{+}^{2}, 0 \leq L_{1}, L_{2} \leq \infty$, be a curve parameterized by arc length. We say that $\gamma$ is minimal if it is smooth, 
$h(\Sigma(\gamma))=0$, and $\operatorname{int}\left(\mathbb{R}_{+}^{2}\right)-\gamma$ has two components, one of them being bounded and containing $N_{\epsilon}$ from above.

Theorem 4.2 (Interior regularity). The apparent horizon is $\Sigma^{*}=\Sigma\left(\gamma^{*}\right)$, where $\gamma^{*}$ (called the horizon curve) is a minimal curve.

Proof. Since $(M, g)$ is $\mathrm{SO}(n+1) \times \mathrm{SO}(m+1)$-invariant it follows that the horizon also is. Therefore, there is a rectifiable curve $\gamma^{*}$ with $\Sigma^{*}=\Sigma\left(\gamma^{*}\right)$, and it follows that $\gamma^{*}$ is bounded since the horizon is compact.

Claim. $\gamma^{*}$ is smooth in the interior of $\mathbb{R}_{+}^{2}$.

Indeed, the apparent horizon outer-minimizes area in the homology class of a marginally trapped hypersurface. Since the mean curvature of the marginally trapped hypersurface is non-positive, a standard argument shows that the horizon is actually a minimizing current. A well-known result of geometric measure theory (see, e.g. [17]) is that the singular set of minimizing currents has codimension 7 or greater. On the other hand, singularities of $\gamma^{*}$ in the interior of $\mathbb{R}_{+}^{2}$ translate into codimension 2 singularities of the horizon $\Sigma^{*}=\Sigma\left(\gamma^{*}\right)$, which proves the claim.

To finalize, recall that a minimizing current is stationary; so wherever the horizon is smooth it must be a minimal hypersurface.

Remark 4.3. Whenever $m+n+2 \leq 7$ the above theorem gives that the horizon is a smooth minimal hypersurface everywhere. If $m, n \leq 6$, the horizon may only have a conical singularity at the origin. Indeed, since the horizon is minimizing it has codimension 7 singularities. If $n+m+2 \leq 7$, the singular set of the horizon can only be empty. For the other case, note that by symmetry the singular set can only have dimension $1, n$ or $m$. This way, if $n, m \leq 6$, the singular set may only be 1-dimensional, so it must be a conical singularity at the origin (see Theorem 4.6 below).

From Equation (3.5) it follows that the condition $h(\Sigma(\gamma))=0$ for minimal curves translates into the following equation for the curve $\gamma(s)=(x(s)$, $y(s))$ :

$$
\kappa+\dot{y}\left(\frac{n}{x}+c \frac{U_{x}}{U}\right)-\dot{x}\left(\frac{m}{y}+c \frac{U_{y}}{U}\right)=0 \quad \text { on } \operatorname{int}\left(\mathbb{R}_{+}^{2}\right) .
$$

Solutions to this equation are graphical almost everywhere with respect to both axes. 
Lemma 4.4. Except at isolated vertical (respectively horizontal) points, a curve that solves (4.1) is locally a graph over the $x$-axis (respectively $y$-axis).

Proof. Suppose $\dot{x}(s)=0$ for all $s \in\left(s_{0}, s_{1}\right)$. Writing Equation (4.1) for $s_{0}<s<s_{1}$ gives $n / x_{0}+c U_{x} / U=0$, where $x_{0}=x\left(\left(s_{1}+s_{2}\right) / 2\right)$. This is impossible since $U_{x} / U$ is never constant on vertical segments. A similar argument works for the horizontal case.

We will show that the apparent horizon is a minimal hypersurface smooth everywhere except possibly at the origin, where it may have a conical singularity. (We remove this last possibility in the next section.) In order to prove this statement we need the following ODE result. Recall that $N_{\epsilon} \subset \mathbb{R}_{+}^{2}$ is the marginally trapped neighborhood of $(0,1)$ from before.

Proposition 4.5. Smooth solutions of Equation (4.1) with an endpoint on the axes minus $N_{\epsilon}$ exist uniquely. This is, if $\gamma$ is smooth and solves Equation (4.1) with $\gamma(0) \in\left(\right.$ axes $\left.-N_{\epsilon}\right)$, then $\gamma$ is unique. Furthermore, $\dot{\gamma}(0)$ is perpendicular to the axis, unless $\gamma(0)=(0,0)$, in which case the angle is $\tan ^{-1} \sqrt{m / n}$. It follows that there exists a neighborhood $O_{\epsilon}$ of the axes inside $\mathbb{R}_{+}^{2}-N_{\epsilon}$ that is foliated by these unique solutions. (See, Figure 1.)

Proof. From Lemma 4.4, solutions of Equation (4.1) with an endpoint on the $y$-axis are graphical over the $x$-axis for a short while. In that case, Equation (4.1) becomes

$$
y^{\prime \prime} /\left(1+y^{\prime 2}\right)+y^{\prime}\left(n / x+c U_{x} / U\right)-\left(m / y+c U_{y} / U\right)=0 .
$$

An ODE analysis of this equation gives local existence of smooth solutions. By inspecting the equation it follows that the condition $y^{\prime}(0)=0$ (or $y^{\prime}(0)=$ $\sqrt{m / n}$ when $y(0)=(0,0))$ is required. The same can be done for solutions that intersect the $x$-axis outside $N_{\epsilon}$.

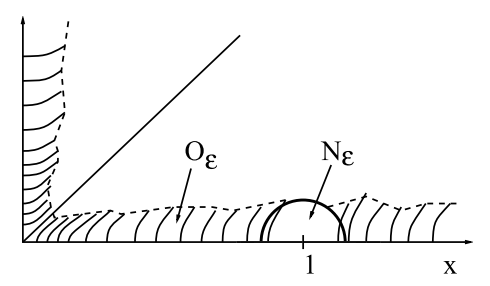

Figure 1: The neighborhood $O_{\epsilon} \subset \mathbb{R}_{+}^{2}-N_{\epsilon}$ is foliated by solutions of (4.1). 
Using the ODE proposition we prove the main result of this section.

Theorem 4.6 (Regularity). The apparent horizon of $(M, g)$ is a minimal hypersurface smooth everywhere except possibly at the origin, where it may have a conical singularity.

Proof. The main idea of the proof is to show that if $\gamma$ is a minimal curve that does not match one of the smooth solution curves that foliate $O_{\epsilon}$ (from Proposition 4.5) as it approaches the boundary, then $\Sigma(\gamma)$ cannot be an area minimizer. We will show this for a curve that has an endpoint in the $y$-axis. The analysis for endpoints on the $x$-axis (outside $N_{\epsilon}$ ) is analogous.

Let $\gamma=(x, y):\left(-L_{1}, L_{2}\right) \rightarrow \mathbb{R}_{+}^{2}$ be a minimal curve. Without loss of generality we analyze the behavior at $L_{2}$ only, where we have $\liminf \operatorname{in}_{s \rightarrow L_{2}}$ $x(s)=0$.

Claim 1. If $\Sigma(\gamma)$ outer-minimizes area in the homology class of a barrier, then $L_{2}<\infty, y(s), x(s)$ are eventually monotonic as $s \rightarrow L_{2}$ and extend continuously to $\left(-L_{1}, L_{2}\right]$.

Indeed, if $\dot{y}\left(s_{i}\right)=0$ for infinitely many $s_{i} \rightarrow L_{2}$, then the curve must be tangent to some leaf of the foliation of $O_{\epsilon}$. By uniqueness of second-order ODEs it follows that $\gamma$ becomes the leaf itself. This gives that $y$ is eventually monotonic and that $\lim _{s \rightarrow L_{2}} y(s)$ exists and is finite. Now suppose $x(s)$ has infinitely many local minima as $s \rightarrow L_{2}$, which are arbitrarily close to the $y$-axis. By cutting off the curve at one of these points (that is close enough to the axis) and replacing that segment by a horizontal line up to the axis, the length of the curve decreases. Furthermore, the cutoff curve continues to enclose the barrier and the area of $\Sigma(\gamma)$ decreases (since the area form is $x^{n} y^{m} U^{2 c} d s$ ). Thus we have found a curve enclosing the barrier with less area than the horizon, but this is impossible since $\Sigma(\gamma)$ outer-minimizes. We deduce that both $x(s), y(s)$ are eventually monotonic. Since the curve is bounded and parameterized by arc length, it follows that $L_{2}$ must be finite. This way, the curve extends continuously to $\left(-L_{1}, L_{2}\right]$ since it is Lipschitz.

From the above argument it follows that $L_{1}, L_{2}<\infty$ and $\gamma$ is continuous on $[0, L]$, where $L=L_{1}+L_{2}$. This proves the claim.

Claim 2. $\gamma$ meets the axes perpendicularly.

From Lemma 4.4, $\gamma$ is locally a graph. In particular, around its endpoint on the $y$-axis, $\gamma$ is the graph of $y(x)$ over the interval $[0, \delta)$ for some $\delta>0$. Assume initially that $y(0)>0$. An analysis of Equation (4.2) gives that 
$y^{\prime}(0)$ exists and is either zero or \pm infinity. If $y^{\prime}(0)= \pm \infty$ then $\gamma$ cannot minimize by a cut-off argument like the one above. This way, it follows that $\Sigma(\gamma)$ is smooth so long as $\gamma$ does not go through the origin. Nevertheless, in that case, we have that

Claim 3. If $\gamma(0)=(0,0)$ then $\gamma$ meets the origin at an angle $\tan ^{-1} \sqrt{m / n}$.

To prove this last part we make a blow-up argument around the origin. Consider the rescaled curve $\gamma_{\lambda}=(u(s), v(s))=(\lambda x(s / \lambda), \lambda y(s / \lambda))$. Multiplying Equation (4.1) by $1 / \lambda$ and evaluating at $s / \lambda$, we get that, in the limit $\lambda \rightarrow \infty$, the rescaled curve solves $\kappa+n \dot{v} / u-m \dot{u} / v=0$. Since this equation is invariant under rescaling of the graph, its solution must be a line. This way $\gamma$ has a tangent line at the origin. A direct calculation shows that this line has an angle $\tan ^{-1} \sqrt{m / n}$.

It is evident that if $\gamma(0)=(0,0)$ the apparent horizon has a singularity at the origin. By the rescaling argument from above we get that the tangent cone of the horizon at the origin is the $\mathrm{SO}(n+1) \times \mathrm{SO}(m+1)$-invariant minimal cone in $\mathbb{R}^{n+m+2}$. This ends the proof.

\section{Topology}

Our goal here is to prove the following result.

Theorem 5.1. The apparent horizon is smooth everywhere and has topology $S^{n} \times S^{m+1}$.

Recall that, from before, the horizon is given by $\Sigma\left(\gamma^{*}\right)$. Furthermore, the horizon is everywhere smooth, unless $\gamma^{*}$ goes through the origin - in that case $\Sigma\left(\gamma^{*}\right)$ gets a conical singularity around the origin.

Idea of the proof of Theorem 5.1. The main idea is to prove that the curve $\gamma^{*}$ is (roughly speaking) a small semicircle around the point $(1,0)$. This way, $\gamma^{*}$ does not go through the origin, and the horizon does not have a conical singularity. Furthermore, we get that the topology of the horizon is automatically $S^{n} \times S^{m+1}$.

In order to show that $\gamma^{*}$ is 'semicircular' we prove that there are three regions in the plane that $\gamma^{*}$ (including its endpoints) must avoid altogether. Please see Figure 2 for a depiction.

Region (1): the marginally trapped region $N_{\epsilon}$ which is a small enough semicircle around $(1,0)$. This region is already forbidden since the end is positive. It exists by the argument in the proof of Theorem 3.5. 


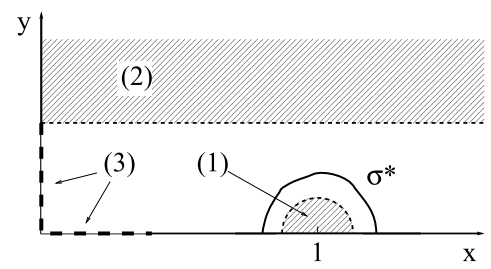

Figure 2: The horizon curve $\gamma^{*}$ and the forbidden regions.

Region (2): the region $\left\{y>c^{\prime} \epsilon^{1 /(n+m)}\right\} \subset \mathbb{R}_{+}^{2}$. This region is forbidden by the maximum principle which we prove in Section 5.1 below. (Here $c^{\prime}>0$ will be a constant independent of $\epsilon$.)

Region (3): a small portion of the axes around the origin. We rule out this region in Section 5.2 below. This portion is forbidden because there is a conical attractor that makes 'almost conical curves' become complete graphs, which are unbounded.

It follows that once regions (1)-(3) are forbidden, all that $\gamma^{*}$ can be is a deformation of a semicircle around $(1,0)$. This is because $\gamma^{*}$ is simple, bounded, and intersects the $x$-axis on each side of $(1,0)$.

\subsection{Maximum principle}

We begin by finding an estimate for the derivatives of $U$ which will be used in the maximum principle.

Lemma 5.2. Let $r^{2}=(x-1)^{2}+y^{2}$ as before. Then

$$
\left|U_{x}\right| \leq \epsilon c^{\prime} r^{-(m+n+1)} \quad \text { and } \quad 0 \leq-U_{y} \leq \epsilon c^{\prime} r^{-(n+m+1)}
$$

where $c^{\prime}>0$ is a constant independent of $\epsilon$.

Proof. From the expansion $U(x, y)=1+\epsilon \int_{S^{n}}\left(y^{2}+\left(x-\xi_{1}\right)^{2}+\xi_{2}^{2}+\cdots+\right.$ $\left.\xi_{n+1}^{2}\right)^{-(n+m) / 2} d \mu(\xi)$, it follows that $\left|U_{x}\right| \leq \epsilon c^{\prime}(x+1) r^{-(m+n+2)}$ and that $0 \leq$ $-U_{y} \leq \epsilon c^{\prime} y r^{-(n+m+2)}$, which gives the result.

Theorem 5.3 (Maximum principle). $\gamma^{*}$ is contained in $\left\{y \leq c^{\prime} \epsilon^{1 /(n+m)}\right\}$, where $c^{\prime}>0$ is a constant independent of $\epsilon$.

Proof. We will show that the maximum height of $\gamma^{*}$ is bounded by $c^{\prime} \epsilon^{1 /(n+m)}$. Indeed, let $\gamma^{*}=(x(s), y(s))$. From the proof of Theorem 4.6 it follows that $y(s)$ has a global maximum at some $s_{0}>0$ for which $\dot{y}\left(s_{0}\right)=0$. We first 
claim that this global maximum is not located on the $y$-axis. Indeed, this follows from the estimates of Lemma 5.10 below, which show that the curve is concave up around the $y$-axis. If the curve is oriented so that $s=0$ at the furthest endpoint on the $x$-axis, then at $s_{0}$ we have $k\left(s_{0}\right) \geq 0$ and $\dot{x}\left(s_{0}\right)=$ -1. Evaluating Equation (4.1) at $s_{0}$ gives $\kappa\left(s_{0}\right)+m / y\left(s_{0}\right)+c U_{y} / U=0$. Using the estimate of Lemma 5.2 together with $U \geq 1$ and $\kappa \geq 0$ we get $m / y\left(s_{0}\right) \leq \epsilon c c^{\prime} r^{-(n+m+1)}$. Since $y \leq r$, it follows that $y\left(s_{0}\right)^{n+m} \leq c c^{\prime} \epsilon / m$. This ends the proof.

\subsection{The conical attractor}

Here we set $\delta=\epsilon^{1 /(n+m+1)}$ to make some expressions simpler. We are interested in proving the following result.

Theorem 5.4. For $\delta>0$ small enough, any curve that solves Equation (4.1) and has an endpoint on $\{0\} \times[0, \delta]$ or on $[0, \delta] \times\{0\}$ is a complete graph, hence unbounded.

We will give a proof of this theorem for curves that have one endpoint on $\{0\} \times[0, \delta]$. The other case is analogous.

Remark 5.5. The depiction of Figure 2 is accurate. Indeed, $(\{0\} \times[0, \delta]) \cup$ $([0, \delta] \times\{0\})$ intersects $\left\{y>c^{\prime} \epsilon^{1 /(n+m)}\right\}$ of the maximum principle since $\delta=$ $\epsilon^{1 /(n+m+1)}$ and $\epsilon^{1 /(n+m+1)}>c^{\prime} \epsilon^{1 /(n+m)}$ for $\epsilon>0$ small enough.

In order to proceed with the proof, we introduce a change of coordinates in Equation (4.2) to study its behavior. Similar coordinates were used by Ilmanen in [12] to study wiggly companions of minimal cones, which satisfy Equation (4.2) with $U \equiv 1$. In that case, the change of coordinates makes the equation an autonomous 2-dimensional system, for which stability analysis is simple. In our case, our equations are not autonomous, so the analysis is more involved.

Consider the coordinates $t=\log x, W=y / x, Z=d y / d x$. Using the chain rule, Equation (4.2) becomes the first-order system

$$
W_{t}(t, W, Z)=Z-W, Z_{t}(t, W, Z)=n \frac{1+Z^{2}}{W}\left(K_{1}-K_{2} W Z\right),
$$

where $K_{1}(t, W)=m / n+c y U_{y} /(n U)$ and $K_{2}(t, W)=1+c y U_{x} / U$.

Let $\gamma$ be a curve that solves Equation (4.1) and has an endpoint on $\{0\} \times[0, \delta]$. Lemma 4.4 gives that $\gamma$ is the graph of a function $y(x)$ that 
solves Equation (4.2) near the axis. Therefore, we may use the $(W, Z)$ coordinates and Equation (5.2) to obtain the 'phase' of $\gamma$, i.e., the tuple $(W, Z)$ that represents $\gamma$ inside of $W Z$-space (at least for a short time) which is given by the above change of coordinates.

Idea of the proof of Theorem 5.4. Equation (5.2) is 'almost' autonomous, which is the motivation for examining the phase plane in the $(W, Z)$ coordinates. (In the Euclidean case, the analogous equation becomes autonomous. See Figure 3(a), and [12].) We show that there exists a trapping region $\Omega$ in the plane, for which the evaluation of the vector field $\left(W_{t}, Z_{t}\right)$ on $\partial \Omega$ always points towards its interior (See Figure 3(b)). Furthermore, we prove that $\Omega$ is bounded in the $Z(=d y / d x)$-direction. This gives that any curve whose phase enters $\Omega$ does not blow up in finite time; therefore it is a complete graph and is unbounded. In the last part of the proof we find global estimates that show that the phase of curves that solve Equation (4.1) and have an endpoint on $\{0\} \times[0, \delta]$ eventually lies in $\Omega$. We can expect this behavior since, in the Euclidean case, a minimal hypersurface that is close to a minimal cone at the origin converges to the cone at infinity. In particular, it remains graphical and is unbounded.

We now introduce the conical attractor, which actually is just an attracting region. (In the Euclidean case, curves in phase space do spiral into the attracting fixed point given by the minimal cone.)

Proposition 5.6. Let $\Omega^{\prime}=\{(\delta, 0) \leq(W, Z) \leq(+\infty, n /(m \delta)+1)\}$ and $C$ as below. Then $\Omega=\Omega^{\prime}-C$ is a trapping region, i.e., the quantity $\left(W_{t}, Z_{t}\right)$ of Equation (5.2) evaluated on $\partial \Omega$ always points towards the inside of $\Omega$.

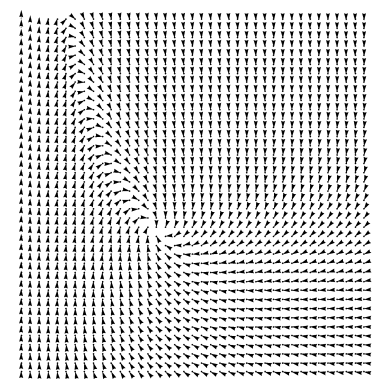

(a)

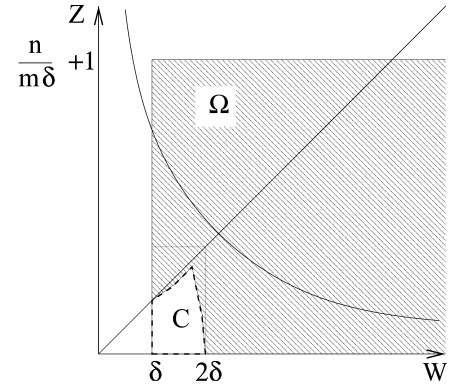

(b)

Figure 3: (a) Direction field $\left(Z_{t}, W_{t}\right)$ for $U=1$. (b) The trapping region $\Omega$. 
The region $C$ is a region bounded by two special curves and two lines. Roughly speaking, $C$ is a 'worst-case scenario region' for the vector field, as we see below. See Figure 3(b) for a depiction of all of them.

We begin by bounding the terms $K_{1}, K_{2}$ from above. We will later use these bounds to construct the worst-case scenario region $C$.

Lemma 5.7. Let $K_{1}, K_{2}$ be as in the system (5.2). On the region $\{(t, W)$ : $W \geq \delta, t \in \mathbb{R}\}$ we have the uniform bounds

$$
\frac{m}{n}-c^{\prime} \epsilon^{\alpha} \leq K_{1}(t, W) \leq \frac{m}{n} \quad \text { and } \quad 1-c^{\prime} \epsilon^{\alpha} \leq K_{2}(t, W) \leq 1+c^{\prime} \epsilon^{\alpha},
$$

where $\alpha=1-(m+n) /(m+n+1)>0$.

Proof. $W \geq \delta$ is just $y \geq \delta x$, so it must be $r \geq \delta$ in that region. The rest follows from applying the estimate (5.1).

A consequence of these estimates are global bounds for the vector field $\left(W_{t}, Z_{t}\right)$ in a particular region of the $W Z$-plane.

Corollary 5.8. Let $R=[\delta, 2 \delta] \times[0,2 \delta]$ and $\left(W_{t}, Z_{t}\right)$ from (5.2). Then

$$
\inf _{\mathbb{R} \times R} W_{t} \geq-2 \delta \quad \text { and } \inf _{\mathbb{R} \times R} Z_{t} \geq \frac{m-n c^{\prime} \epsilon^{\alpha}}{2 \delta}-\delta\left(1+c^{\prime} \epsilon^{\alpha}\right)\left(2 n+8 \delta^{2}\right),
$$

where $\alpha=1-(m+n) /(m+n+1)>0$.

The set $C$ is constructed as follows. Let $\sigma$ denote an integral curve of $\left(W_{t}, Z_{t}\right)$ in the $W Z$-plane that starts at the bottom right corner of $R$, i.e., at the point $p=(2 \delta, 0)$. (Note: such a curve exists by general ODE results, but it is not necessarily unique since the system (5.2) is not autonomous.) Let $R_{1}(\sigma) \subset R$ denote the region inside $R$ that is bounded on top by the diagonal and bounded on the right by the curve $\sigma$. We define $C_{1}$ to be the intersection of all the possible $R_{1}(\sigma)$, where $\sigma$ is an integral curve that starts at $p$. (This is, $C_{1}$ is a worst-case scenario region for integral curves that start at $p.) C_{1}$ is not empty. Indeed, a direct calculation using Corollary 5.8 shows that

Lemma 5.9. Let $L$ be the line $Z(W)=-W\left(m / 4 \delta^{2}\right)+m / 2 \delta^{2}$. Then $C_{1}$ includes the region $R_{1}(L)$, and $R_{1}(L) \supset(\{\delta\} \times[0, \delta] \cup[\delta, 2 \delta] \times\{0\})$.

Now let $\sigma$ denote an integral curve that starts at $q=(\delta, \delta)$, and let $R_{2}(\sigma)$ denote the region inside $C_{1}$ bounded on top by the curve $\sigma$. Note that any 
such $\sigma$ is increasing (as a graph on the $W$-axis) since $W_{t}$ is positive inside $R$. We define $C$ to be the intersection of all the possible $R_{2}(\sigma)$, where $\sigma$ is an integral curve that starts at $q$. (This is, add to $C_{1}$ a worst-case scenario for integral curves that start at q.) $C$ is not empty since $\sigma$ is increasing. Indeed, we are, at worst, capping off the top of $C_{1}$ by a horizontal line through $q$. See Figure 3(b).

Proof of Proposition 5.6. The boundary of $\Omega$ consists of five parts as depicted in Figure 3(b). The roof is given by a segment of the line $\{Z=$ $n /(m \delta)+1\}$ that lies well inside the region $Z=K_{1} /\left(K_{2} W\right)$. This gives that $Z_{t}$ points downward on the top. The bottom boundary is part of the positive $W$-axis, and $Z_{t}$ is positive there. The left-side boundary consists of three segments. One is the segment of the line $\{W=\delta\}$ that lies above the diagonal, for which $W_{t}$ is non-negative. What is left is the top and right of the boundary of $C$. From the construction it follows that $\left(W_{t}, Z_{t}\right)$ points inwards there as well.

We now show that the phase of any curve that solves Equation (4.2) and has an endpoint on $\{0\} \times[0, \delta]$ eventually lies in $\Omega$. In order to do that, we compute a second-order approximation of these solutions. We check that after a small time they are close to the conical solution. (See Figure 1 for a snapshot of this phenomenon.)

Lemma 5.10. A smooth solution of Equation (4.2) with $y(0)>0$ satisfies

$$
y^{\prime \prime}(0)=\frac{m}{(n+1)} \frac{1}{y(0)}+c \frac{U_{y} / U-U_{x} / U}{n+1}=\frac{m}{(n+1)} \frac{1}{y(0)}+\epsilon O\left(r^{-(m+n+1)}\right) .
$$

Proof. We know from the proof of Theorem 4.6 that $y^{\prime}(0)=0$. Multiplying (4.2) by $y$, taking limit $x \rightarrow 0$ and using L'Hopital's rule in the term $y^{\prime} / x$ gives $(n+1) y^{\prime \prime}(0) y(0)-m+y(0) c\left(U_{x} / U-U_{y} / U\right)=0$. Together with the gradient estimate of Lemma 5.2 and the fact that $U \geq 1$, this gives the result.

Corollary 5.11. Suppose y solves Equation (4.2) with $y(0)=\delta$ for $\delta>0$ small enough. Then for $0 \leq x \leq \delta$, we have that

$$
y(x)=\delta+\frac{m}{2(n+1) \delta} x^{2}+O(\epsilon), \quad \text { and } \quad y^{\prime}(x)=\frac{m x}{(n+1) \delta}+O(\epsilon) .
$$

Proof. Follows from Lemma 5.10 and Taylor's theorem. 
Proposition 5.12. For $\delta \geq 0$ small enough, the phase of any solution $y$ of Equation (4.2) with $y(0)=\delta$ eventually enters the region $\Omega$.

Proof. We first prove this for a curve with $y(0)=0$. Indeed, in that case $y(x)=\sqrt{m / n} x+O(\epsilon)$ and $y^{\prime}(x)=\sqrt{m / n}+O(\epsilon)$, from before. This way, for small $x>0$ we get $W=y / x \approx \sqrt{m / n}$ and $Z=y^{\prime} \approx \sqrt{m / n}$, so $(W, Z)$ lies inside $\Omega$.

Whenever $y(0)=\delta>0$ is small, using the expansions of Corollary 5.11 evaluated at $x=\delta$ gives $W=y(\delta) / \delta \approx 1+m / 2(n+1)$ and $Z=y^{\prime}(\delta) \approx m /(n+$ $1)$, which also gives a point that lies within $\Omega$.

Proof of Theorem 5.4. Follows directly from the above proposition together with Proposition 5.6. This ends the proof of Theorem 1.1 as well.

Corollary 5.13. $(M, g)$ contains a non-compact minimal hypersurface with a conical singularity at the origin.

Proof. Follows directly from Proposition 4.5 together with Theorem 5.4.

\section{Acknowledgments}

I would like to thank Hubert Bray for his encouragement and many useful discussions. I would also like to thank Bill Allard, Greg Galloway, Bob Wald and Brian White for their useful conversations.

\section{References}

[1] R. Bartnik, The mass of an asymptotically flat manifold, Comm. Pure Appl. Math. 39(5) (1986), 661-693.

[2] E. Bombieri, E. De Giorgi and E. Giusti, Minimal cones and the Bernstein problem, Invent. Math. 7 (1969), 243-268.

[3] L. Caffarelli, R. Hardt and L. Simon, Minimal surfaces with isolated singularities, Manuscripta Math. 48(1-3) (1984), 1-18.

[4] H. Elvang and P. Figueras, Black saturn, Preprint, 2007, available at hep-th/0701035.

[5] R. Emparan and H.S. Reall, A rotating black ring solution in five dimensions, Phys. Rev. Lett. 88(10) (2002), 101101, 4. 
[6] R. Emparan, T. Harmark, V. Niarchos, A.O. Niels and M.J. Rodriguez, The phase structure of higher-dimensional black rings and black holes Preprint, 2007, available at arXiv:0708.2181[hep-th].

[7] G.J. Galloway, Rigidity of outer horizons and the topology of black holes, Preprint, 2006, available at gr-qc/0608118.

[8] G.J. Galloway and R. Schoen, A generalization of Hawking's black hole topology theorem to higher dimensions, Comm. Math. Phys. 266(2) (2006), 571-576.

[9] D. Gilbarg and N.S. Trudinger, Elliptic partial differential equations of second order, Classics in Mathematics, Springer-Verlag, Berlin, 2001. Reprint of the 1998 edition.

[10] B. Gidas, W.M. Ni, and L. Nirenberg, Symmetry and related properties via the maximum principle, Comm. Math. Phys. 68(3) (1979), 209-243.

[11] G. Huisken and T. Ilmanen, The inverse mean curvature flow and the Riemannian Penrose inequality, J. Differential Geom. 59(3) (2001), 353-437.

[12] T. Ilmanen, Lectures on mean curvature flow and related equations, 1998. Draft version.

[13] W.H. Meeks III and S.T. Yau, Topology of three-dimensional manifolds and the embedding problems in minimal surface theory, Ann. of Math. (2) $\mathbf{1 1 2}(3)$ (1980), 441-484.

[14] W. Meeks III, L. Simon, and S.T. Yau, Embedded minimal surfaces, exotic spheres, and manifolds with positive Ricci curvature, Ann. of Math. (2) 116(3) (1982), 621-659.

[15] A.W. Peet, TASI lectures on black holes in string theory, Preprint, 1999, available at hep-th/0008241.

[16] R. Schoen and S.T. Yau, On the structure of manifolds with positive scalar curvature, Manuscripta Math. 28(1-3) (1979), 159-183.

[17] L. Simon, Lectures on geometric measure theory, Proceedings of the Centre for Mathematical Analysis, Australian National University, Vol. 3, Australian National University Centre for Mathematical Analysis, Canberra, 1983.

[18] - Lectures on singularities of variational problems, Tsing Hua Lectures on Geometry \& Analysis (Hsinchu, 1990-1991), 1997, pp. 279-298. 
[19] M. Struwe, Variational methods, 2nd edn, Ergebnisse der Mathematik und ihrer Gren-zgebiete (3) [Results in Mathematics and Related Areas (3)], vol. 34, Springer-Verlag, Berlin, 1996. Applications to nonlinear partial differential equations and Hamiltonian systems.

[20] R.M. Wald, General relativity, University of Chicago Press, Chicago, IL, 1984.

Mathematics Department

DUKE UNIVERSITY

DURhAM NC 27708, USA

Current address:

Mathematics Institute

ZeEMAN BUILDing

UNIVERSITY OF WARWICK

Coventry CV4 7AL, UK

E-mail address: f.a.schwartz@warwick.ac.uk

Received June 14, 2007 
